\title{
Economic Diverging Diamond Interchange (DDI) Design for Reducing Traffic Signal Timings and Easy Pedestrian Crossing
}

\author{
Muhammad Saleem
}

\begin{abstract}
Usually traffic signal timings are almost 95 seconds per signal (in 4-way intersection). In this paper we apply the Diverging Diamond Interchange (DDI) idea on the city traffic intersection. DDI's are built over freeway overpass (or underpass). But instead of building overpass or underpass which is economically not feasible for Pakistan) we just apply DDI concept on city traffic intersection on ground level to reduce signal timings, cost for building DDI, greater safety for pedestrians and ease of passing for ambulances and other emergency services are also discussed in this paper.
\end{abstract}

Keywords---traffic signal timings, intersection, pedestrian.

\section{INTRODUCTION}

Prior to 2009 the only known diverging diamond interchanges were in France in the communities of Versailles, Le Perreux-sur-Marne and Seclin, all built in the 1970s.[1] (The ramps of the first two have been reconfigured to accommodate ramps of other interchanges, but they continue to function as diverging diamond interchanges.)

Despite the fact that such interchanges already existed, the idea for the DDI was "reinvented" around 2000, inspired by the former "synchronized split-phasing" type freeway-tofreeway interchange between Interstate 95 and I-695 north of Baltimore.[2].

The concept of DDI will cost too much especially for developing country like Pakistan. And also building a underpass or overpass (bridge) to control traffic jams and congestion would cost millions of dollars with already tough economic times of Pakistan.

The problem of traffic jams at intersections are causing many problems such as delays in appointments, long lines at traffic signals and even deaths if ambulance get trapped inside traffic jams.

So, alternate solution should be followed to solve this problem. And the proposed design must be optimized in order to satisfy all requirements while minimizing the cost. The idea is to provide DDI type assembly by removing the portion for freeway overpass or underpass and use the assembly for the city traffic. By this it will reduce traffic signal timing. And also 2 way emergency lanes are also provided for rapid movement of emergency services like ambulances. Pedestrians will also find it easy to cross because this type of assembly consists of almost 13 zebra crossings.

Muhammad Saleem, Wah Engineering College, University of Wah, Pakistan.
This type of project requires a little more space than a normal intersection. But it will reduce traffic jams without building under pass or bridge. We spend spent millions of dollars in building overpasses and underpasses then why don't we just use portion of that money to clear little space and make it traffic passage more smooth and economically feasible.

\section{WHAT IS DIVERGING DIAMOND INTERCHANGE (DDI)?}

A diverging diamond interchange (DDI), also called a double crossover diamond interchange (DCD), [3] is a type of diamond interchange in which the two directions of traffic on the non-freeway road cross to the opposite side on both sides of the bridge at the freeway. It is unusual in that it requires traffic on the freeway overpass (or underpass) to briefly drive on the opposite side of the road from what is customary for the jurisdiction. The diverging diamond interchange was listed by Popular Science magazine as one of the best innovations in 2009 (engineering category) in "Best of What's New 2009".[4]

Like the continuous flow intersection, the diverging diamond interchange allows for two-phase operation at all signalized intersections within the interchange. This is a significant improvement in safety, since no long turns (e.g. left turns where traffic drives on the right side of the road) must clear opposing traffic and all movements are discrete, with most controlled by traffic signals.[5] 


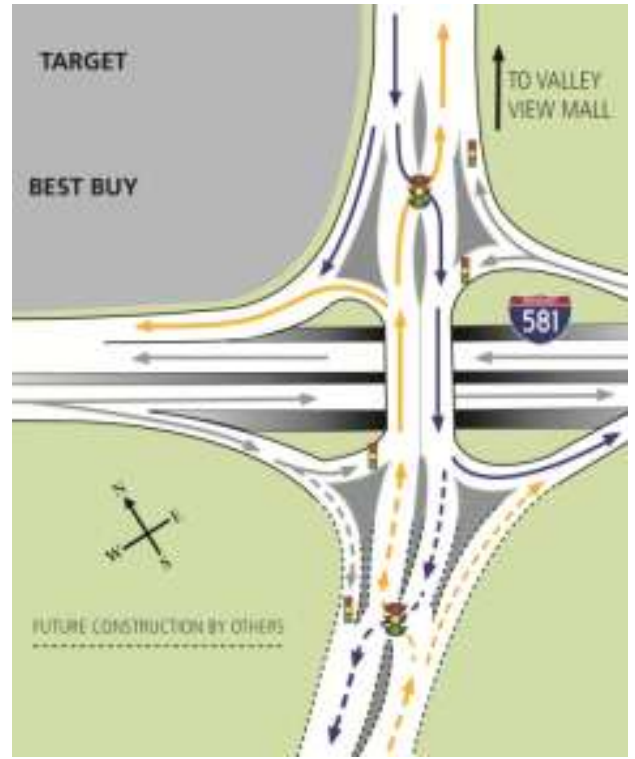

Fig. 1: Valley View Diverging Diamond Interchange Concept, State of Virginia, United States.

\section{How Much IT Costs?}

The United State's first diverging diamond, which opened in Springfield, Mo., in 2009. The original plan for expanding that intersection was set to cost $\$ 10$ million, but the diverging diamond ended up costing only $\$ 3.2$ million.

In other words DDI can save us millions of dollars if it to be used as interchange.

\section{Why Do We NeEd ThIS TYPE OF CONFIGURATION FoR TRAFFIC INTERSECTIONS?}

As traffic is increasing day by day and so are the traffic congestion and traffic jams on the signals especially in fast developing countries like China. This creates negative impact over both economy and people.

Traffic congestion has many effects. One of the most important is parking problems. People find it difficult to park their cars especially in the city center. Long delays in getting to and from work are another result, leading to less productivity from employees. There are also more accidents, because people become frustrated or angry due to the traffic jams. Pollution in the city center worsens as a result of car emissions, and the city becomes a less attractive place to live. Shops are forced to close in the city center and have to relocate to malls or to the outskirts of the city, where parking is available.[6]

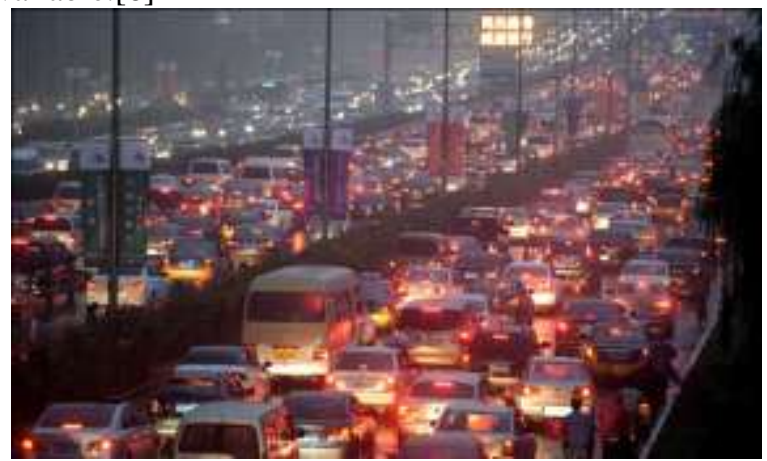

Fig. 2: A traffic jam on a street in Beijing in September 2014.[7]
Many developed countries have built very confusing intersection bridges like Nanpu Bridge in Shanghai China (see figure 3) to reduce traffic jams. Shanghai Nanpu Bridge costs almost $227 \$$ millions.

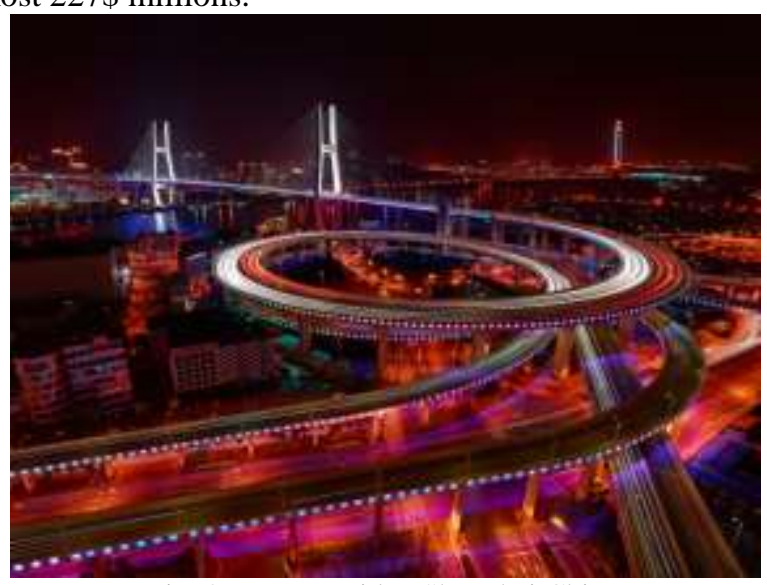

Fig. 3: Nanpu Bridge Shanghai China

Other countries like Saudi Arabia have built many underpasses to reduce the travel time. And also to get rid of traffic jams.

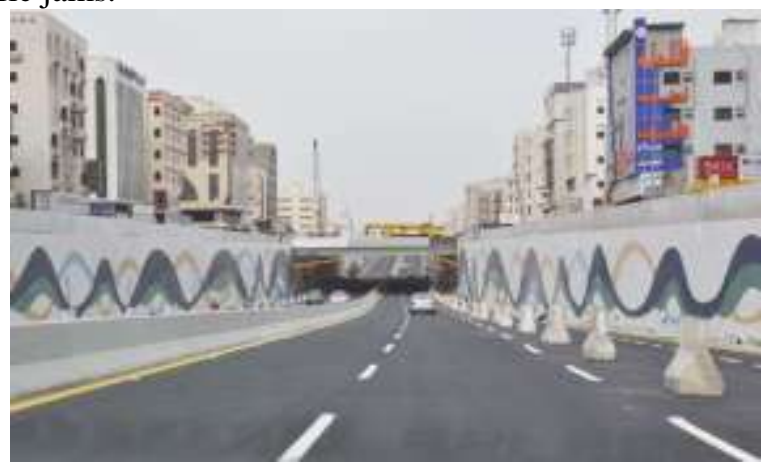

Fig. 4: Travel time from Sitten Bridge to Sabaeen Street now takes just a few minutes with the opening of Tahlia-Makarona underpass in Jeddah Saudi Arabia

Estimate cost per square feet for building overpass (Over roadway) is $\$ 145$ and almost same is for building underpass. Both of the techniques for reducing traffic jams can work but the cost for building them is very high for developing country like Pakistan. So, we must follow a cheaper and more economical way to solve this problem.

\section{V.PROPOSED IDEA}

Proposed idea is to keep the configuration of the junction same as the Diverging Diamond interchange. In other words just transform the Diverging Diamond interchange into special type of traffic intersection without building any kind of overpass or underpass to make it simple, save and economical. 


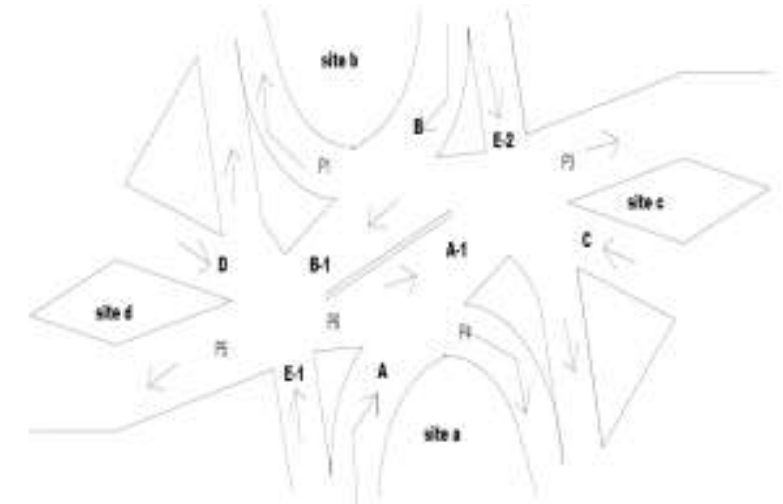

Fig. 5: Shows the proposed idea of the intersection.

In figure 5 signals are marked as A, A-1, B, B-1, C and D. Signals opening and closing configuration is same as for Diverging Diamond interchange has. But this special intersection doesn't have freeways overpass or underpass. Instead it has paths marked as E-1 and E-2 these are the emergency lanes that are discussed in next sections.

\section{TRAFFIC Signals CONFIGURATIONS}

As discussed earlier traffic signal opening and closing configuration is same as of Diverging Diamond interchange (DDI).

Signal at D and C open (green) (for almost say 30 seconds) at the same time for the traffic and traffic can follow the following paths shown in figure 6 . And signal at A, A-1, B and B-1 is close (red) (for almost say $34 \mathrm{sec}$ ).

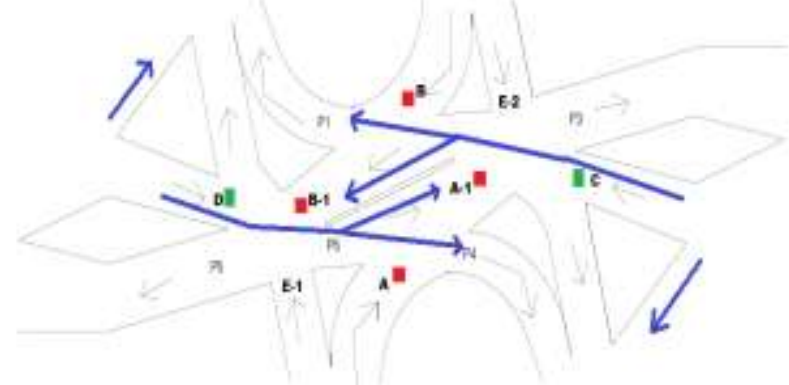

Fig. 6: Blue lines shows the path, green box indicates that traffic signal is open and red box indicates that traffic signal is close.

When signal at D and $\mathrm{C}$ is closed (red) (for almost say 34 sec) then the signal at A, A-1, B and B-1 is open (green) (for almost say 30 seconds) at the same time for the traffic and traffic can follow the following paths shown in figure 7.

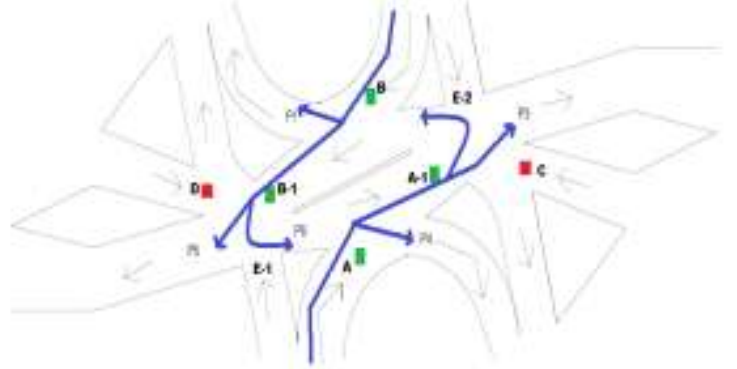

Fig. 7: Blue lines shows the path, green box indicates that traffic signal is open and red box indicates that traffic signal is close.
In this way traffic has to wait only 34 seconds or 64 seconds rather than 90 or even 95 seconds for green light. So by this configuration traffic is controlled without additional costs for overpasses or underpasses.

\section{EMERGENCY LANE}

Marked E-1 and E-2 in figure 5 are the emergency lanes as discussed earlier. These lanes have instant excess for movement only for emergency services like ambulances. Because if they want to travel straight from point A to point P-1 it has to travel long way and waste a lot of time.

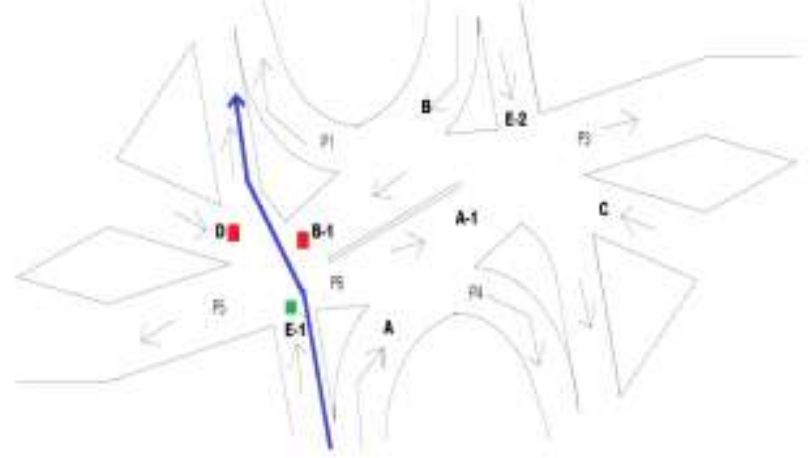

Fig. 8: Blue lines shows the path, green box indicates that traffic signal is open and red box indicates that traffic signal is close.

Moreover another benefit of this emergency lane it has sensor to detect vehicle. Once ambulance (for example) has entered this lane it turns green and turns adjacent signals red. For example it is using lane E-1 (see figure 8) then signal at E-1 turns green and signal at B-1 and D turns red automatically through sensor. Allowing ambulance to pass smoothly without interruption or delays.

\section{PEDESTRIAN FRIENDLY}

Road accidents are increasing every day. People crossing the busy roads despite the running vehicles have become a common phenomenon. This puts many liabilities on both the people as well as the drivers. In most cases the drivers are helpless. Instead of using the pedestrian bridge, people jaywalk across the busy roads just to save a few minutes. As soon as the signal turns green drivers hasten to drive their car while people are also in a hurry to cross the roads. Accidents frequently occur at such times. People are not conscious while crossing. [8]

This type of intersection as shown in figure 5 has 13 zebra crossings. By this pedestrian find it easy to without traffic congestion. Moreover, pedestrian have to wait less for their turn to cross roads. But traveling time may increase because they have to travel through at least 4 zebra crossings. But they have greater safety while crossing because they have shorter zebra crossing than larger of normal traffic signals.

\section{LIGHTING BY SOLARPANELS}

Solar panels placement requires empty spaces which are usually insufficient in normal intersections. Also it requires poles to place them or just placed on street lights but they are not sufficient for traffic lights and its functions. Empty spaces at site a, site b, site $\mathrm{c}$ and site d (see figure 5) can be used for 
placing on ground with certain arrangements for protection from weather and security.

\section{X.ADVANTAGES}

1. It will increase the attractiveness of intersection because if overpass bridge or underpass have been built instead then it will decrease the attractiveness of that place.

2. Two-phase signals with short cycle lengths, significantly reducing delay.

3. Reduced horizontal curvature reduces risk of offroad crashes.

4. Increases the capacity of turning movements to and from the ramps.

\section{DisadVantages}

1. Drivers may not be familiar with configuration, particularly with regards to merging maneuvers along the left side of the roadway or the crossover flow of traffic.

2. It requires a little more empty space for construction than normal traffic intersection but less space than overpass or underpass.

3. As it requires clear space for its construction so it requires additional cost to clear the land.

\section{FURTHER CONSIDERATIONS}

$>$ No standards currently exist for this design

$>$ The design depends on site-specific conditions.

\section{XIII.CONCLUSION}

Cost saving is the biggest advantage of this project. It can provide cheaper solution to traffic jams and also to reduce traffic signal timings.

It is to be kept in mind this type of configuration is best suitable where there is already very empty space. And also this can provide revolutionary changes in intersection construction.

Before building such kind of intersection we must first aware drivers about how to use this type of intersection.

We spend spent millions of dollars in building overpasses and underpasses then why don't we just use portion of that money to clear little space and make it traffic passage more smooth and economically feasible.

\section{REFERNCES}

[1] Staff (June 13, 2013). "I-64 Interchange at Route 15, Zion Crossroads". Virginia Department of Transportation. Retrieved December 11, 2013.

[2] Chlewicki, Gilbert (2003). "New Interchange and Intersection Designs: The Synchronized Split-Phasing Intersection and the Diverging Diamond Interchange" (PDF). Retrieved 2009-10-20.

[3] Hughes, Warren; Jagannathan, Ram (October 2009). "Double Crossover Diamond Interchange". Federal Highway Administration. FHWA-HRT09-054. Retrieved April 22, 2012.

[4] "Gallery: Looking Back at the 100 Best Innovations of 2009". Popular Science. Retrieved January 20, 2012.

[5] "Diverging Diamond Interchange". OHM Advisors.

[6] http://writefix.com/?page_id=808

[7] http://www.theguardian.com/global/2015/jun/01/smog-noodles-chinapollution-injustices-achievements-tania-branigan
[8] Muhammad Saleem, 2015, 'New ways of building pedestrian flyover bridge', 1st Multi-Disciplinary Student Research Conference (MDSRC2015), 14-15 November 2015, University of Wah (UW), Wah cantt Pakistan, pp. 5 